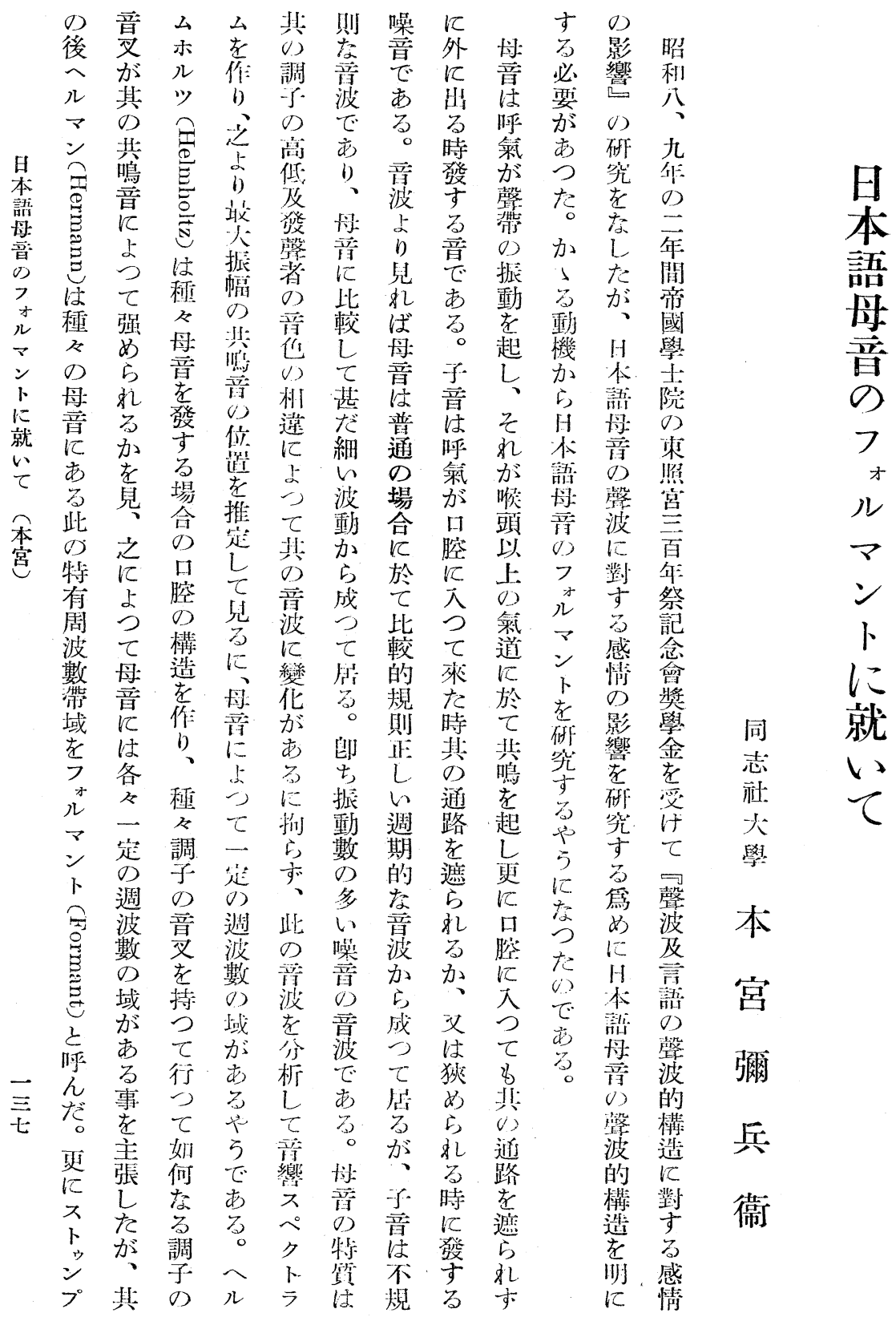




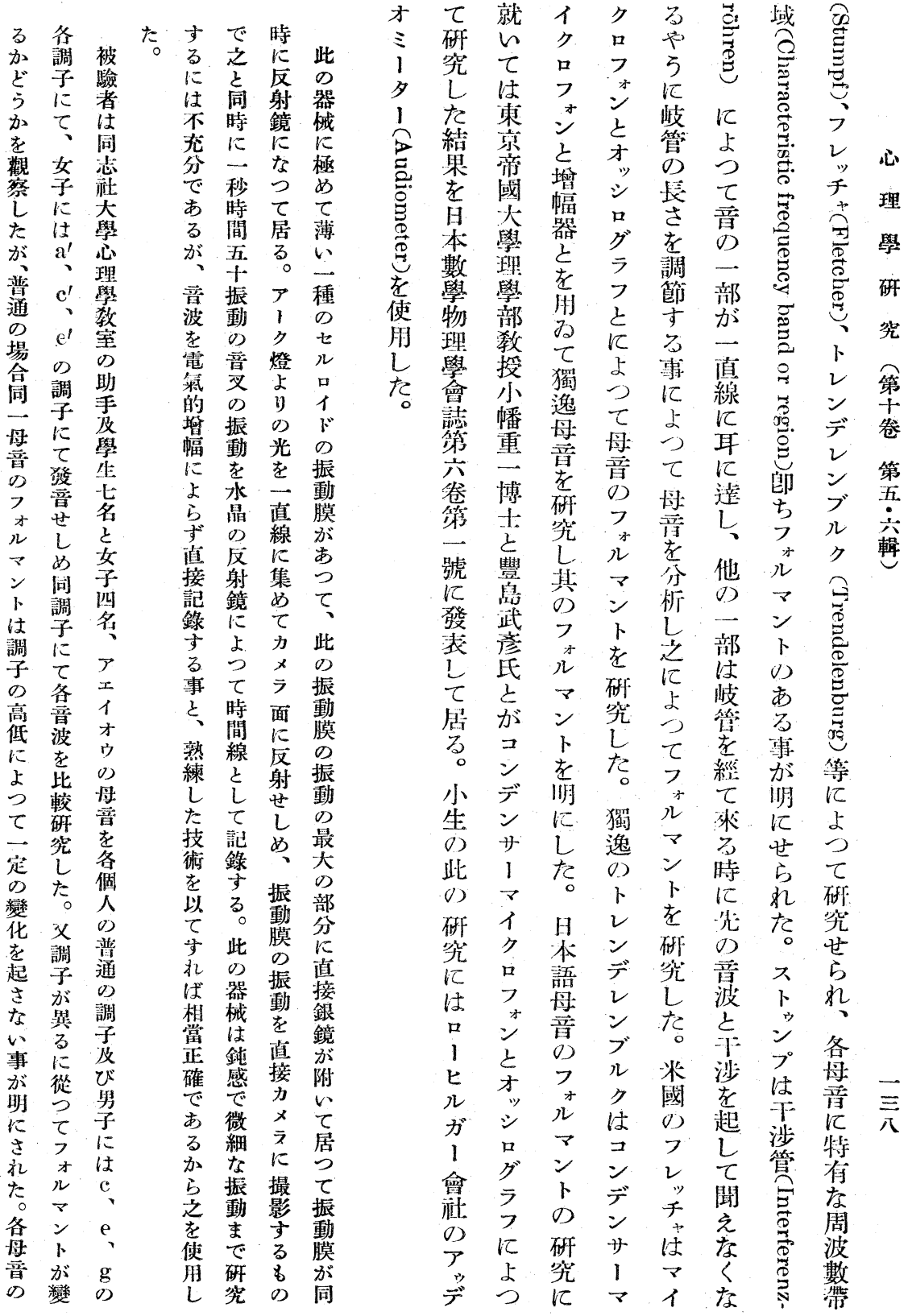


母音アのフォルマント(罗聲)

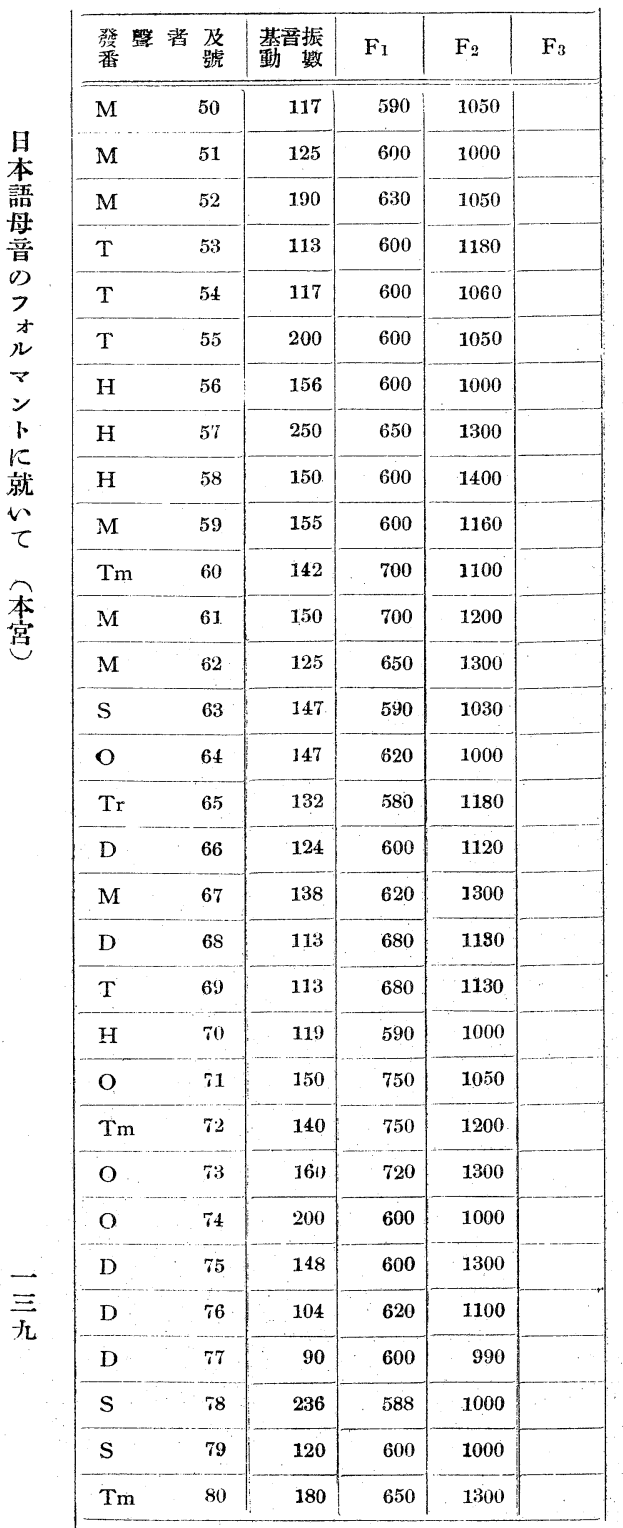

小次㵒

阔少域

前 表

音 示

屬打

屬长

万标

亿音

为順

丸 順

口序

我は

閐音

奥 馨

母: 學

音 0

份

屬 類

于

万從 動

才哓 數

及 正

び日に

丸大多

而閐 $心$ 仵

牛同 偣

開 意

奥 音

丹江 0 音

音屬最 0

守大域

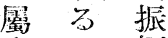

寸 ア幅

る、孝

ウ非有

(日)

順坐疗

ᄀ 數

序開 倍 为帶

に前音儿域

從 母 $\sigma$ ?

音域 ン

事量的

K 屬 $\mathrm{F}_{3}$ "I

寸 寸 奌

万存特

工推 份

定 周

官

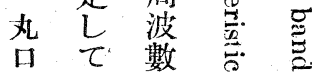

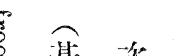

基 次

允富

是 27

近等 $\begin{gathered}\text { 基 } \\ \text { i }\end{gathered}$

振音基么

動 響竟に

數 倍

○ ○ 意 影

倍ク更

音, 關に

關 摴

釉 娎

0) 公最

最

大表

大 悬

倍 器

振占意火

幅り 怘よ

D各 位 $y$

範之置 約

䁶第支

二盟蓓

K摭

、ル し 势

管

管 V

才 II

儿低

マ 特

○准

下周

II 波哭

中㜢諘

特

和

分

㭕

各

()

波

y
誳
分
析
法

K

$\stackrel{5}{=}$

?

分

蛋分

产

: 


(つ
\begin{tabular}{|ll|l|l|l|}
\hline $\mathrm{S}$ & 151 & 147 & 290 & \\
\hline $\mathrm{O}$ & 152 & 130 & 260 & \\
\hline $\operatorname{Tr}$ & 153 & 133 & 260 & \\
\hline $\mathrm{D}$ & 154 & 123 & 250 & \\
\hline
\end{tabular}

\begin{tabular}{|c|c|c|c|c|c|}
\hline $\mathrm{M}$ & 81 & 150 & 750 & 1050 & \\
\hline $\mathrm{Tm}$ & 82 & 118 & 700 & 1060 & \\
\hline $\mathrm{M}$ & 83 & 276 & 600 & 1100 & \\
\hline $\mathrm{O}$ & 84 & 262 & & 1050 & 3000 \\
\hline $\mathrm{M}$ & 101 & 125 & 620 & 1130 & \\
\hline $\mathrm{M}$ & 102 & 160 & 680 & 1060 & \\
\hline$M$ & 103 & 188 & 600 & 1060 & \\
\hline $\mathrm{O}$ & $10 t$ & 126 & 700 & 1250 & \\
\hline $\mathrm{O}$ & 10.5 & 158 & 590 & 1400 & \\
\hline $\mathrm{O}$ & 106 & 186 & $60 \%$ & 990 & \\
\hline
\end{tabular}

\begin{tabular}{|c|c|c|c|c|c|}
\hline \multicolumn{2}{|c|}{ 發嘿者及番號 } & 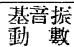 & $F_{i}$ & $\mathrm{~F}_{2}$ & $\mathrm{~F}_{3}$ \\
\hline M & 183 & 120 & 400 & 940 & $*$ \\
\hline M & 181 & 159 & 410 & & 1380 \\
\hline $\mathbf{M}$ & 182 & 191 & 440 & 850 & 1910 \\
\hline $\mathrm{O}$ & 186 & 127 & 500 & 870 & 1390 \\
\hline $\mathrm{O}$ & 187 & 156 & 570 & 980 & $14 \therefore 80$ \\
\hline O & 188 & 183 & 470 & 900 & 1290 \\
\hline $\mathrm{S}$ & 184 & 200 & 480 & 1000 & 1900 \\
\hline M & 186 & 126 & 400 & 930 & * \\
\hline $\mathrm{s}$ & 187 & 152 & 490 & 760 & 1300 \\
\hline $\mathrm{O}$ & 189 & 129 & 500 & 900 & 1400 \\
\hline $\operatorname{Tr}$ & 185 & 120 & 480 & 1000 & 1520 \\
\hline $\mathrm{D}$ & 190 & 125 & 400 & 1000 & 1320 \\
\hline
\end{tabular}

母音ウのフォルマント(男㢣)

\begin{tabular}{|c|c|c|c|c|c|}
\hline \multicolumn{2}{|c|}{ 發㢣者及番號 } & $\begin{array}{l}\text { 基音振 } \\
\text { 數 }\end{array}$ & $F_{1}$ & $\mathrm{~F}_{2}$ & $\mathrm{~F}_{3}$ \\
\hline M & 161 & 128 & 410 & 1420 & \\
\hline $\mathrm{M}$ & 162 & 159 & 390 & 1000 & \\
\hline $\mathrm{M}$ & 163 & 188 & 440 & 1000 & \\
\hline $\mathrm{O}$ & 165 & 128 & 350 & 1090 & \\
\hline $\mathrm{O}$ & 166 & 158 & 450 & 1330 & \\
\hline $\mathrm{O}$ & 167 & 186 & 430 & 1100 & 2000 \\
\hline $\mathbf{M}$ & 168 & 128 & 380 & 1000 & \\
\hline $\mathrm{S}$ & 364 & 152 & 460 & 1300 & 2200 \\
\hline $\mathrm{O}$ & 169 & 130 & 350 & 1000 & \\
\hline $\operatorname{Tr}$ & 163 & 133 & 350 & 1060 & \\
\hline D & 170 & 127 & 300 & 1370 & \\
\hline
\end{tabular}

母音エのフォルマント (罗㢣)

\begin{tabular}{|c|c|c|c|c|}
\hline 發警者及番號 & 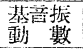 & $F_{1}$ & $F_{2}$ & $F_{3}$ \\
\hline 171 & 159 & 550 & 1500 & \\
\hline
\end{tabular}

\begin{tabular}{ll|lll|l}
\hline $\mathrm{M}$ & 172 & 191 & 430 & 1910 & \\
\hline $\mathrm{M}$ & 173 & 128 & 650 & 1280 & \\
\hline $\mathrm{S}$ & 174 & 151 & 480 & 1660 & \\
\hline $\mathrm{Tr}$ & 175 & 123 & 490 & 1300 & \\
\hline $\mathrm{O}$ & 176 & 125 & 560 & 1310 & \\
\hline $\mathrm{O}$ & 177 & 157 & 540 & 1730 & \\
\hline $\mathrm{O}$ & 178 & 186 & 440 & 1600 & \\
\hline $\mathrm{O}$ & 179 & 130 & 350 & 1350 & \\
\hline $\mathrm{D}$ & 98 & 125 & 370 & 1280 & \\
\hline $\mathrm{M}$ & 99 & 250 & 370 & 1300 &
\end{tabular}

沚音イのフォルマント(男㢣)

\begin{tabular}{|c|c|c|c|c|c|}
\hline \multicolumn{2}{|c|}{ 發警掏及乘號 } & $\begin{array}{l}\text { 基涪振 } \\
\text { 锥力數 }\end{array}$ & $\mathrm{F}_{1}$ & $F_{2}$ & $\mathrm{~F}_{3}$ \\
\hline M & 131 & 126 & 250. & & \\
\hline M & 132 & 159 & $300^{\circ}$ & & \\
\hline M & 133 & 183 & 350 & & \\
\hline $\mathrm{O}$ & 134 & 128 & 260 & & \\
\hline $\mathrm{O}$ & $1: 5$ & 157 & 300 & $(1720)$ & \\
\hline $\mathrm{O}$ & 136 & 192 & 350 & & $(2100)$ \\
\hline M & 150 & 113 & 250 & & \\
\hline
\end{tabular}


母音ウのフォルマント(女㢣)

\begin{tabular}{|c|c|c|c|c|c|}
\hline \multicolumn{2}{|c|}{ 發馨者及番號 } & 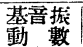 & $F_{1}$ & $\mathrm{~F}_{3}$ & $F_{2}$ \\
\hline Ms & 21 & 259 & * & 1560 & 2850 \\
\hline Ms & 22 & 334 & $\%$ & 1550 & 2340 \\
\hline Ms & 23 & 394 & $*$ & 1900 & 2980 \\
\hline $\mathrm{U}_{\mathrm{m}}$ & 24 & 319 & $*$ & 1590 & 3600 \\
\hline $\mathrm{Sr}$ & 25 & 322 & $*$ & 1500 & 2800 \\
\hline
\end{tabular}

日本語母竟フォルマント(男馨)

\begin{tabular}{|c|c|c|c|c|}
\hline 拇音 & 分析件數 & $F_{1}$ & $\mathrm{~F}_{2}$ & $\mathrm{~F}_{3}$ \\
\hline 3 & 41 & $580-750$ & $990-1400$ & 3000 \\
\hline$x$ & 11 & $350-650$ & $1280-1910$ & $*$ \\
\hline 1 & 11 & $250-350$ & & $*$ \\
\hline オ & 12 & $400-570$ & $760-1000$ & $1290-1910$ \\
\hline ウ & 11 & $390-460$ & $1000-1420$ & $2000-2200$ \\
\hline
\end{tabular}

\begin{tabular}{|c|c|c|c|c|}
\hline 挑䈏 & 分析件數 & $F_{1}$ & $\mathrm{~F}_{2}$ & $\mathrm{~F}_{3}$ \\
\hline$\gamma$ & 5 & $1020-1160$ & $1600-\div 360$ & $2800-3530$ \\
\hline$x$ & 5 & 550 & $1200-1820$ & $2250-2800$ \\
\hline 1 & 5 & (600以下) & & $2230-3160$ \\
\hline オ & 5 & $500-630$ & $830-1030$ & $2300-3000$ \\
\hline ウ & 5 & $*(500$ 以下) & $1500-1900$ & $2340-360$ \\
\hline
\end{tabular}

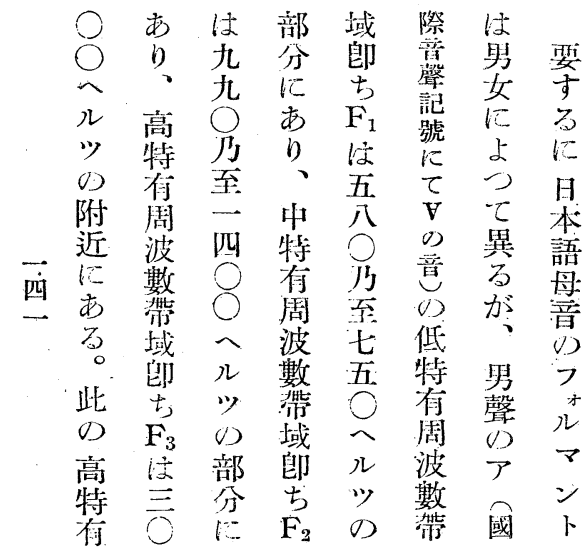

母音アのフォルマント(女㢣)

\begin{tabular}{|c|c|c|c|c|c|}
\hline \multicolumn{2}{|c|}{ 發㹂者及番號 } & $\begin{array}{l}\text { 基音振 } \\
\text { 數 }\end{array}$ & $F_{1}$ & $\mathrm{~F}_{2}$ & $F_{3}$ \\
\hline Ms & 121 & 255 & 1020 & 2100 & 2800 \\
\hline Ms & 122 & 320 & 1090 & 2360 & 3400 \\
\hline $\mathrm{M}_{\mathrm{s}}$ & ¿ 23 & 384 & 1150 & 1680 & 3530 \\
\hline Um & 124 & 330 & 1160 & $16 \div 0$ & 3300 \\
\hline $\mathrm{Sh}$ & 125 & 319 & 1070 & 1600 & 2900 \\
\hline
\end{tabular}

母音エのフォルマント(女㢣)

\begin{tabular}{|c|c|c|c|c|c|}
\hline \multicolumn{2}{|c|}{ 發聲者及番號 } & $\begin{array}{l}\text { 基曋癄 } \\
\text { 動 數 }\end{array}$ & $F_{1}$ & $F_{2}$ & $\mathrm{~F}_{3}$ \\
\hline Ms & 31 & 259 & 550 & $*$ & 2270 \\
\hline Ms & 32 & 325 & * & 1700 & 2430 \\
\hline $\mathrm{Ms}_{\mathrm{s}}$ & 33 & 330 & $*$ & 1820 & 2700 \\
\hline $\mathrm{Um}_{\mathrm{m}}$ & 34 & 316 & $*$ & 1200 & 2800 \\
\hline $\mathrm{Sr}$ & 35 & 320 & $\because$ & 1200 & 2250 \\
\hline
\end{tabular}

母湆イのフォルマント(女馨)

\begin{tabular}{|c|c|c|c|c|c|}
\hline \multicolumn{2}{|c|}{ 發擎老及㸌號 } & 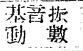 & $F_{1}$ & $\mathrm{~F}_{2}$ & $\mathrm{~F}_{3}$ \\
\hline Ms & 137 & 263 & $*$ & & 2230 \\
\hline $\mathrm{Ms}$ & 138 & 327 & $*$ & 2020 & 3160 \\
\hline $\mathrm{Ms}$ & 138 & 392 & $*$ & & 2740 \\
\hline $\mathrm{Um}$ & 140 & $3: 0$ & $\%$ & & 3000 \\
\hline $\mathrm{Sr}$ & 141 & 350 & $\because$ & & 2900 \\
\hline
\end{tabular}

母意オのフォルマント(女聲)

\begin{tabular}{|c|c|c|c|c|c|}
\hline \multicolumn{2}{|c|}{ 紧馨者及番躆 } & 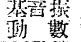 & $F_{1}$ & $\mathrm{~F}_{2}$ & $\dot{F}_{3}$ \\
\hline $\mathrm{Ms}$ & 41 & 255 & 500 & 830 & 2300 \\
\hline Ms & 42 & 31. & 630. & 1000 & 2860 \\
\hline Ms & 43 & 388 & $*$ & $8 \% 0$ & 2300 \\
\hline $\mathrm{Um}$ & 44 & 326 & $*$ & 1000 & 3000 \\
\hline $\mathrm{Sr}$ & 4.) & 325 & 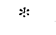 & 1030 & 2950 \\
\hline
\end{tabular}


析 0

日本語母吾のフォルマント(男㛑)

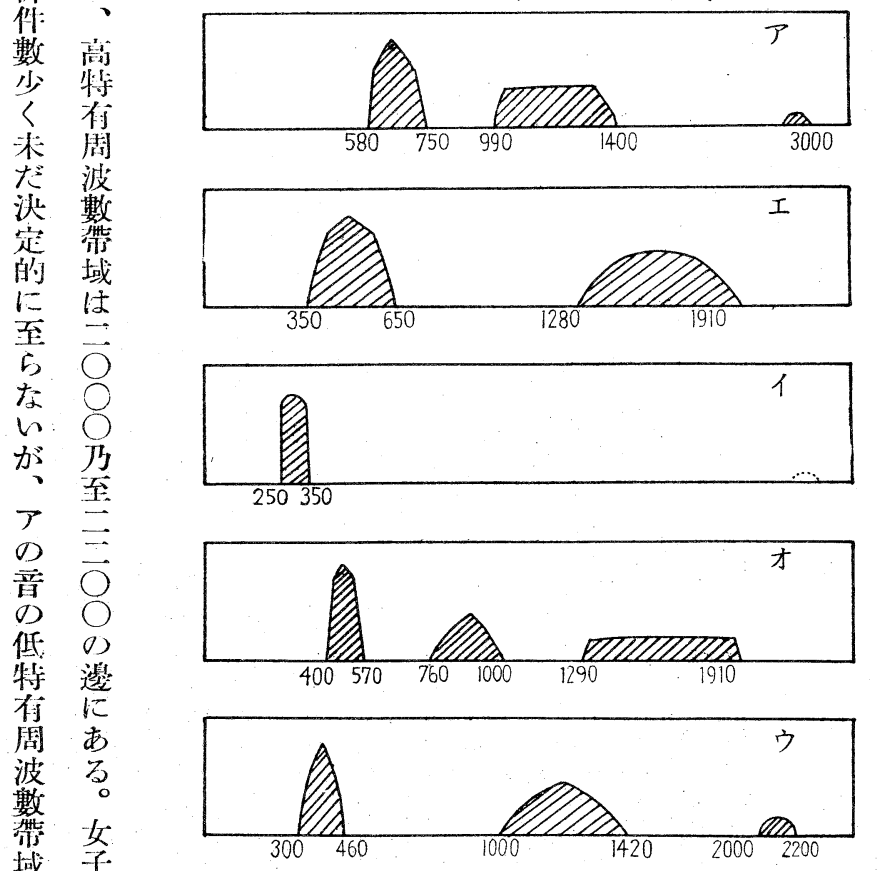

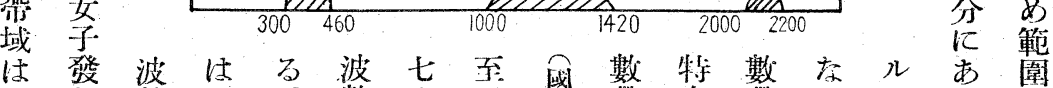

二馨 數 三數 六五際帶们帶加ツ り を

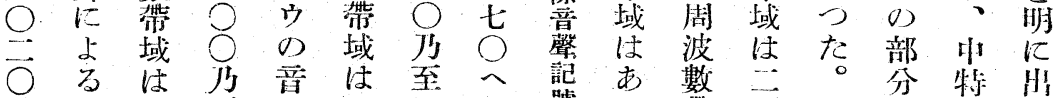

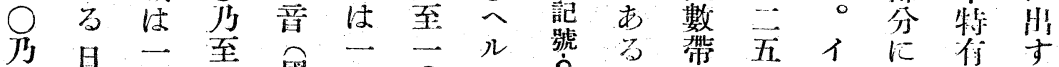

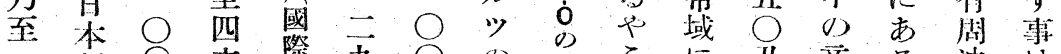

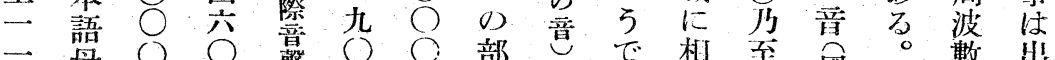

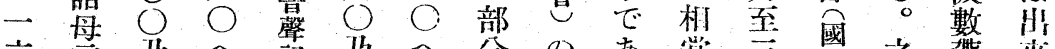

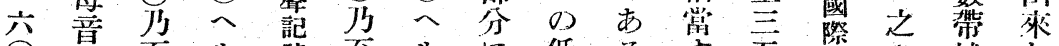

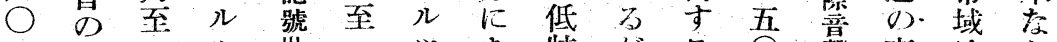

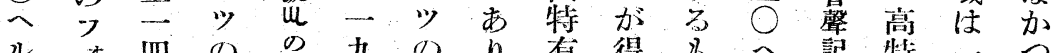

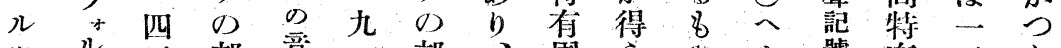

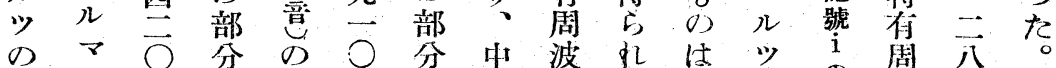

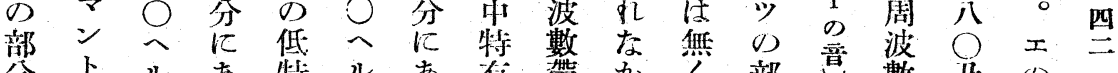

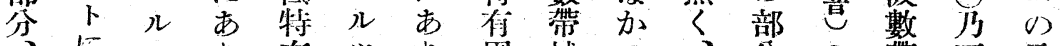

、に り 有 $ッ$ 、周域

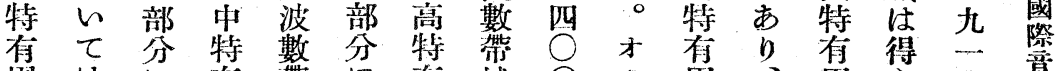

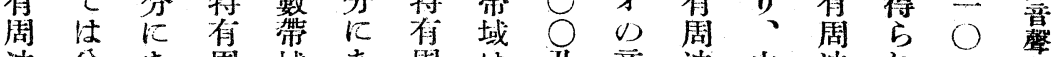

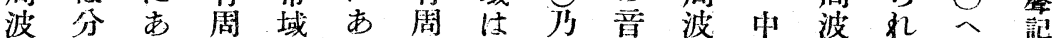


大愈にて な

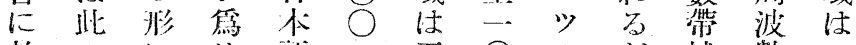

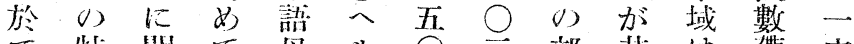

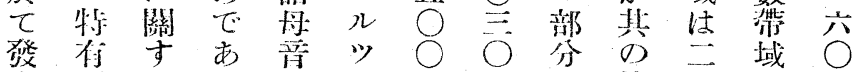

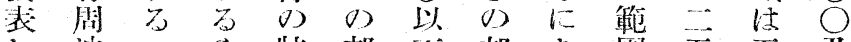
上波一き特部卡部古国五五出

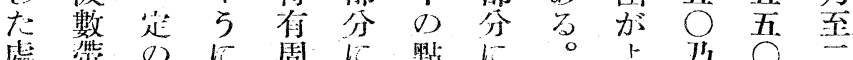

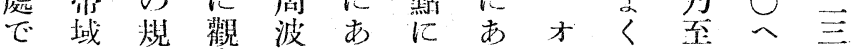
あに定察數 り

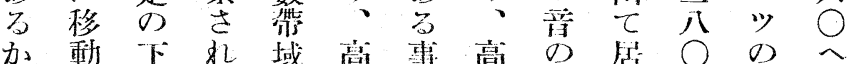
らかた る 竝起發。倠有み有特なのに に名馨被人周知周虍心部あ心 尛。驗に波方波缃。分り部

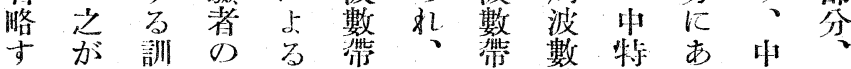
号感練 開範 域 其域帶 信号特 高 情が口園はのは域周。份特 の大支の範三は波イ周倠 種々度移 三圍三五數の波風

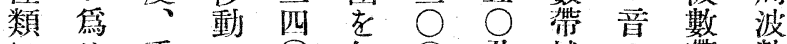
にめ活の $\bigcirc$ 知 $\bigcirc$ 出域 の覍數 占での 比而号至の低域帶 つあ高 較 至事至六部特は域 てるさ的三が至分有一性

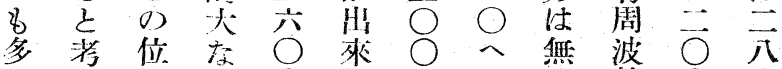

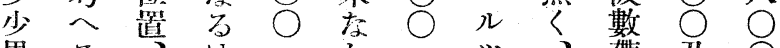

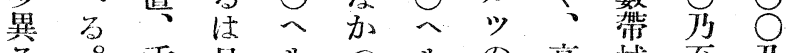
弓。 舌日 事义の 术 は同前語の。の分有六八 昭し後音 部中部に風 $\bigcirc$ 五

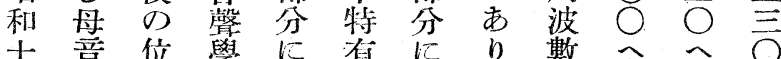

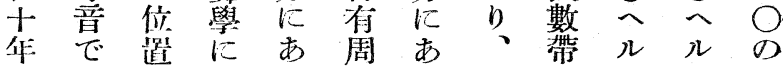

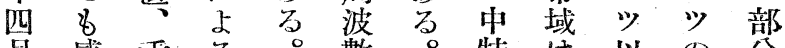
月感舌方。數。特は以 0 分

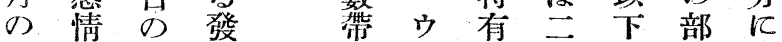
日 形聲 域の周三の分あ

本多、法

は音波言點にる

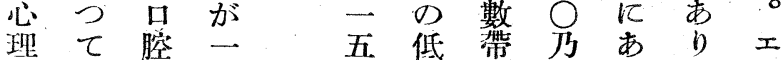

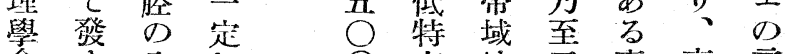
會守全 $\bigcirc$ 有は主事高音

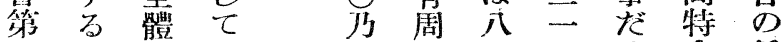

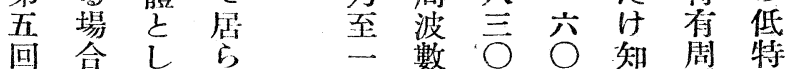


(From the Psychological Institute, Doshisha-University)

\title{
On the Characteristic Frequency Bands of the Japanese Vowels
}

\author{
(Abstract) \\ by

\section{Y. Motomiya}

The Low-Hilger Audiometer was used to describe the vocal waves of the Japanese vowels in this study. The subjects of this experiment are seven males among the assistants and students of the psychological laboratory of the Doshisha University and four females. They were demanded to pronounce $\mathrm{r}$ з $\mathrm{i}$ o $\mathrm{u}$ of Japanese vowels in their ordinary speaking tones and these were recorded. Then the male subjects were demanded to pronounce the same vowels in different tones of $\mathrm{c}$, e and $\mathrm{g}$ and the female subjects were demanded to pronounce in $\mathrm{a}^{\prime} ; \mathrm{c}^{\prime}$ and $\mathrm{e}^{\prime}$. These vocal waves were analysed by the Fourier harmonic analysis on the 24-ordinate scheme. Acoustic Spectrums were made by the results of these analyses. The frequency of the maximum resonance, that is, the pitch of the characteristic frequency region was estimated by connecting the highest points of each spectrum. These frequencies of the maximum resonance of each vowel were summed up and thus the characteristic frequency bands of the Japanese vowels were decided in the following.

The characteristic frequency bands of the Japanese vowels (male)

\begin{tabular}{|cc|c|c|c|c|}
\hline \multicolumn{2}{|c|}{ Vowel } & Cases analysed & F1 & F2 & F3 \\
\hline $\mathrm{V}$ & $\nearrow$ & 41 & $580-750$ & $990-\mathbf{1 4 0 0}$ & 3000 \\
\hline 3 & $\simeq$ & 11 & $350-650$ & $1280-1910$ & $*$ \\
\hline $\mathrm{i}$ & $\nearrow$ & 11 & $250-350$ & & $*$ \\
\hline $\mathrm{o}$ & $オ$ & 12 & $400-570$ & $760-1000$ & $1290-1910$ \\
\hline $\mathrm{u} x$ & $ウ$ & 11 & $300-460$ & $1000-1420$ & $2000-2200$ \\
\hline
\end{tabular}


(female)

\begin{tabular}{|c|c|c|c|c|c|}
\hline & Vowel & Cases analysed & F1 & F2. & F3 \\
\hline$y$ & $\Gamma$ & 5 & $1020-1160$ & $1600-2360$ & $2800-35330$ \\
\hline 3 & $I$ & 5 & 550 & $1200-1820$ & $2250-2800$ \\
\hline i & 1 & 5 & $*($ less than 600$)$ & & $2230-3160$ \\
\hline$\dot{0}$ & オ & 5 & $500-630$ & $830-1030$ & $2300-3000$ \\
\hline & ウ & 5 & $*(1$ ess than 500) & $1500-1900$ & $2340-3600$ \\
\hline
\end{tabular}

* = This indicates that the spectrum was too short to decide the region

F1 = The lower characteristic frequency band

F $2=$ The middle characteristic frequency baud

F3 = The higher characteristic frequency band 